\title{
Pilot study of intratumoral (IT) cryoablation (cryo) in combination with systemic checkpoint blockade in patients with metastatic melanoma (MM)
}

\author{
Dae Won Kim", Cara Haymaker ${ }^{2}$, Natalie McQuail², Elizabeth Sirmans², Christine Spencer ${ }^{2}$, Isabella Glitza², \\ Rodabe Amaria², Scott Woodman², Sapna Patel ${ }^{2}$, Michael Davies², Cassian Yee², Wen-Jen Hwu², \\ Chantale Bernatchez², Jennifer Wargo², Padmanee Sharma², James Allison², Patrick Hwu², Alda Tam², Adi Diab²
}

From 30th Annual Meeting and Associated Programs of the Society for Immunotherapy of Cancer (SITC 2015) National Harbor, MD, USA. 4-8 November 2015

\section{Background}

Cryo is an effective modality for pain palliation and local control of soft tissue and bone metastases. Cryo induces necrotic cell death, and combination cryo with antiCTLA-4 generates potent systemic anti-tumor immune responses in preclinical models and small clinical studies. However, the clinical activity of cryo plus checkpoint blockade has not been evaluated in MM. In this pilot study, we report the safety and efficacy of IT cryo and systemic ipilimumab or pembrolizumab in patients with MM.

\section{Methods}

A single-institution study of $16 \mathrm{MM}$ patients who received IT cryo during systemic therapy of checkpoint blockade. Pts with at least 1 symptomatic lesion amenable for cryo were included. Cryo was performed under ultrasound or CT-guidance. Data of symptom control, toxicity assessment and responses were collected prospectively and continued after 12 weeks of treatment administration. Exploratory immune correlates from peripheral blood and tumor samples were obtained when available.

\section{Results}

12 out of 16 treated pts are evaluable at this time. Median age was 60 years (range: 58-82). All pts received cryo after 1 or 2 doses of either ipilimumab $(\mathrm{N}=8)$ or pembrolizumab $(\mathrm{N}=4) .8$ patients had BRAF-V600 mutation and 3 had NRAS mutation. 10 pts had stage-IV disease (M1a: 2, M1b: 1, M1c: 7) and 2 had unresectable stage III. 4 pts were treatment naïve and 8 received 1-5 prior treatments.

${ }^{1}$ Moffitt, Tampa, FL, USA

Full list of author information is available at the end of the article
Objective response rates (ORR) of cryoablated (local) lesions were $75 \%$ (9/12), ORR of distant lesions were $40 \%$ $(4 / 10)$ and total ORR (local+distant) were 50\% (2 [with local lesions only] +4 [distant] $=6 / 12$ ) (ipilimumab-63\% (5/8), pembrolizumab-25\% (1/4)) by RECIST 1.1. Local disease control rates (DCR) were $83 \%$, distant DCR were $60 \%$, and overall DCR were $75 \%$. With a median followup of 7 months, the progression-free survival rate at 6 months was $57 \%$. At the time of the analysis, the median progression-free survival and overall survival have not been reached. Grade 3 immune related (irAE) toxicities were observed in 2 patients including colitis and hypophysitis. There were no other grade $3 / 4$ irAEs or toxicities related to cryo. Immunological analysis is ongoing.

\section{Conclusions}

The study results suggest that the combination of cryo with checkpoint blockades is safe, well-tolerated and could potentially be an effective strategy to enhance the anti-tumor activity. These findings, warrant further evaluation in a larger prospective trial.

\section{Authors' details}

'Moffitt, Tampa, FL, USA. ${ }^{2}$ The University of Texas MD Anderson Cancer Center, Houston, TX, USA.

Published: 4 November 2015

doi:10.1186/2051-1426-3-S2-P137

Cite this article as: Kim et al:: Pilot study of intratumoral (IT) cryoablation (cryo) in combination with systemic checkpoint blockade in patients with metastatic melanoma (MM). Journal for ImmunoTherapy of Cancer 2015 3(Suppl 2):P137. 